\title{
Drug distribution and clinical safety in treating cystic craniopharyngiomas using intracavitary radiotherapy with phosphorus-32 colloid
}

\author{
HONGBO CHANG, JIANNING ZHANG, WEIDONG CAO, YAMING WANG, \\ HULIN ZHAO, RUI LIU and SHENGLI GUO
}

Institute of Neurosurgery, Navy General Hospital, Beijing 100048, P.R. China

Received February 4, 2017; Accepted January 8, 2018

DOI: $10.3892 / \mathrm{ol} .2018 .7981$

\begin{abstract}
The present study evaluated drug distribution and clinical safety in treating patients with cystic craniopharyngioma $(\mathrm{CP})$ with intracavitary radiotherapy using phosphorus-32 $\left({ }^{32} \mathrm{P}\right)$ colloid. In total, 40 patients who were recently diagnosed with primary or recurrent cystic $\mathrm{CP}$ were enrolled into the study. Patients underwent stereotactic intracavitary therapy and were administered ${ }^{32} \mathrm{P}$ colloid and iopamidol-300 (1:1 dilution). Head computed tomography (CT) scans were performed $2 \mathrm{~h}$ after surgery in order to assess drug distribution and leakage. Results obtained from the ophthalmic examination (visual acuity, visual field and fundus), enhanced head magnetic resonance imaging and/or CT scans, blood analysis, coagulation tests, electrolyte tests, pituitary hormone level analysis, and hepatic and renal function tests were compared between the $0.5,1,1.5$ and $2 \mathrm{mCi}$ groups. The ${ }^{32} \mathrm{P}$ colloid per minute radioactive count was quantitatively measured in urine and blood samples using a CAPRAC well-type NaI $\gamma$ counter at 1, 3 and 7 days post-surgery. In total, 6, 2 and 1 case(s) from the $2,1.5$ and $1 \mathrm{mCi}$ groups, respectively, demonstrated heterogeneous drug distribution and intracavitary cerebrospinal fluid leakage. Furthermore, out of 24 patients, no significant differences were identified in blood analysis, blood biochemical measurements and pituitary hormone levels prior to and 7 days after surgery. Blood ${ }^{32} \mathrm{P}$ deposition returned to normal levels within 3 days after surgery, whereas urine deposition returned to normal within 7 days after surgery. Methods utilized in the present study were advantageous in terms of convenience, speed and low cost, therefore, these techniques are suitable for continuous monitoring of patient ${ }^{32} \mathrm{P}$ colloid deposition.
\end{abstract}

Correspondence to: Professor Jianning Zhang, Institute of Neurosurgery, Navy General Hospital, 6 Fucheng Road, Beijing 100048, P.R. China

E-mail: jnzhang2005@163.com

Key words: craniopharyngioma, drug distribution, phosphorus-32, safety

\section{Introduction}

Therapeutic regimens utilized for recurrent craniopharyngioma (CP) primarily comprise repeat surgery, radiotherapy and stereotactic intracavitary therapy (radiotherapy or chemotherapy) (1). In 1985, Taasan et al (2) reported on 6 patients (3 with CP and 3 with glioma) who were treated with stereotactic intracavitary brachytherapy using phosphorus-32 $\left({ }^{32} \mathrm{P}\right)$ colloid. Results demonstrated that all patients experienced an improved clinical curative effect. Furthermore, the standard clinical ${ }^{32} \mathrm{P}$ colloid dosage (2) based on the size of the cystic cavity was proposed. In 2004, Hasegawa et al (3) reported on 49 CP patients with a mean cyst volume of $13 \mathrm{ml}$. All patients were treated with stereotactic ${ }^{32} \mathrm{P}$ intracavitary irradiation. A radiation dose beween 189 and 250 Gy (mean, 224 Gy) was targeted at the cyst wall during five half-lives of the isotope. The survival rates were $90 \%$ at 5 years and $80 \%$ at 10 years after initial diagnosis. The tumor cyst control rates were $76 \%$ at 5 years and $70 \%$ at 10 years after initial diagnosis. In 2012, Kickingereder et al (4) summarized the application of stereotactic ${ }^{32} \mathrm{P}$ colloid irradiation in 53 patients with cystic CP: Use of a capsule volume of 0.5-78.9 ml, assuming a capsule wall dose of 200-250 Gy, a ${ }^{32} \mathrm{P}$ colloid dose of 0.03-78.9 $\mathrm{mCi}$ and pull-out fluid at $0-29.3 \mathrm{ml}$, and follow-up following affirmation of the curative effect. The application of ${ }^{32} \mathrm{P}$ colloid for radiotherapy on the treatment of cystic CP using stereotactic surgery is a key topic of interest within the Institute of Neurosurgery (Navy General Hospital, Beijing, China) (5,6). According to the current literature, studies on intracavitary drug distribution, surgical leakage and ${ }^{32} \mathrm{P}$ deposition in treating patients with $\mathrm{CP}$ using intracavitary radiotherapy with ${ }^{32} \mathrm{P}$ colloid are limited in number. Furthermore, to the best of our knowledge, there are currently no studies on the monitoring of cerebrospinal fluid (CSF) and blood parameters following ${ }^{32} \mathrm{P}$ colloid treatment for patients with $\mathrm{CP}(2,3)$. In 2012, Trippel and Nikkhah (7) summarized previous literature on the use of stereotactic intracavitary radionuclide therapy for patients with CP and suggested that drug distribution prior to and following radionuclide injection should be compared in order to avoid leakage or connection to the ventricular system; otherwise fever, headache, hypothalamic radiation injury and other effects may occur. Therefore, a safe and convenient technique is required to monitor the distribution of ${ }^{32} \mathrm{P}$ injected into 
the CP cavity, and to detect whether leakage is present. In 2013, Denis-Bacelar et al (8) performed single-photon emission computed tomography (CT) imaging pre- and post-surgery and identified that the mean absorbed dose of ${ }^{32} \mathrm{P}$ colloid delivered to the cyst wall was $<50 \%$ of the total dose and outlined the requirement for further studies on the drug metabolism, residual deposition and safety of ${ }^{32} \mathrm{P}$ for patients with $\mathrm{CP}$.

\section{Materials and methods}

\section{Clinical data}

Primary drugs and equipment. In total, $300 \mathrm{mgI} / \mathrm{ml}{ }^{32} \mathrm{P}$ colloid (Atom Hi-tech Co. Ltd., Beijing, China) and iopamidol-300 (Shanghai Bracco Sine Pharmaceutical Corp., Ltd., Shanghai, China) were injected into the $\mathrm{CP}$ cystic cavity of each patient (1:1 dilution) one time. ${ }^{32} \mathrm{P}$ is a sterile and green colloidal solution with a nuclear purity $>99.9 \%$, $(\mathrm{pH} 7.0)$, a chemical concentration of $2.32 \mathrm{mg} / \mathrm{ml}$ and a radioactive concentration $>1,850 \mathrm{mBq} / \mathrm{ml}$. A CAPRAC well-type $\mathrm{NaI} \gamma$ counter (Beijing Huaruison Science and Technology Development Co. Ltd., Beijing, China) was used to quantitatively measure the ${ }^{32} \mathrm{P}$ colloid per minute radioactive count.

Subjects and grouping. Patients who were recently diagnosed with primary or recurrent cystic CP were enrolled into the study between March 2012 and October 2015. Only patients who were not allergic to iodine and required surgical intervention were enrolled as study subjects. Written informed consent was obtained from either the patient or a relative, and the present study was approved by the Ethics Committee of the Navy General Hospital. In total, 40 patients (26 men and 14 women) aged between 10 and 65 years of age (mean age, 34.3) were selected. The equation for calculating the dose/volume [previously described by Taasan et al (2)] was referred to for the tailored calculation of therapeutic doses [in milliCurie (mCi)] based on clinical and surgical requirements. For the analysis of drug distribution, patients were divided into the following four groups according to therapeutic dose: $0.5 \mathrm{mCi}(0.2 \mathrm{ml} ; \mathrm{n}=10)$, $1 \mathrm{mCi}(0.4 \mathrm{ml} ; \mathrm{n}=10), 1.5 \mathrm{mCi}(0.6 \mathrm{ml} ; \mathrm{n}=10)$ and $2 \mathrm{mCi}(0.8 \mathrm{ml}$; $\mathrm{n}=10)$. Additionally, 8 patients from all groups, with the exception of the $1.5 \mathrm{mCi}$ group (as a result of the little effect of the drug dose between 1.0-2.0 mCi on the experimental results of drug safety study) were randomly selected for an evaluation of drug safety through comparison of hematological parameters prior to and following surgery among different groups and comparison of residue ${ }^{32} \mathrm{P}$ depositions in venous blood and urine among different groups. Due to the large number of specimens (13 $\mathrm{ml}$ preoperative blood samples, $13 \mathrm{ml}$ blood samples from 7 days after surgery, $2 \mathrm{ml}$ blood samples from 1, 3 and 7 days after surgery, $2 \mathrm{ml}$ urine samples from 1, 3 and 7 days after surgery) required from each patient in this part of the study, in order to ensure the smooth progress of the experiment (a smaller number of patients would be easier to monitor because the drug safety analysis required 7 days in hospital following surgery), the number of cases included in the drug safety analysis was reduced.

\section{Therapeutic methods}

Surgery. Local anesthesia was adopted for surgery. The Leksell head frame was used for all patients preoperatively. The positioning magnetic resonance imaging (MRI) scan was performed, and the acquired images were transferred to the stereotactic imaging workshop for artificial puncture route planning and the calculation of cystic cavity volume and drug dose. The Aero Tech stereotactic surgery planning system (supplied by the Image Center of Beihang University, Beijing, China) was used to determine the surgical target and calculate coordinates, whereas the three-dimensional simulation was adopted to determine the optimal surgical approach that should avoid important structures, including blood vessels and optic nerves. During the surgery, ${ }^{32} \mathrm{P}$ colloid was administered for the stereotactic intracavitary therapy in a 1:1 dilution with iopamidol 300 (300 mgI/ml). Drug distribution was assessed using head CT scans within $2 \mathrm{~h}$ after surgery.

Physical examination and imaging examination. Ophthalmic examination (visual acuity, visual field and fundus) and head MRI or CT examinations were performed prior to and following surgery.

Hematological examination. Prior to and 7 days after surgery, the following haematological parameters were assessed for each patient: Blood routine, prothrombin time, activated partial thromboplastin time, thrombin time, fibrinogen, serum total protein, serum albumin, alanine aminotransferase (ALT), aspartate aminotransferase, alkaline phosphatase, creatinine, blood urea nitrogen, potassium ion $\left(\mathrm{K}^{+}\right)$levels, sodium ion $\left(\mathrm{Na}^{+}\right)$levels, chloride ion $\left(\mathrm{Cl}^{-}\right)$levels and pituitary hormones [including thyroid-stimulating hormone (TSH), adrenocorticotropic hormone (ACTH), luteinizing hormone ( $\mathrm{LH})$, follicle-stimulating hormone, prolactin (PRL) and growth hormone $(\mathrm{GH})]$. Patients were require to fast for $10 \mathrm{~h}$ prior to blood sampling $(13 \mathrm{ml})$. Patients who demonstrated a decrease in hormone levels were administered hormone supplements (including thyroxine tablets, dexamethasone tablets and ambroxol tablets) and regular follow-ups (the first week, the first month, the third month and the half year after discharge) were performed the major hematological parameters (ALT, AST, $\mathrm{K}^{+}, \mathrm{Na}^{+}, \mathrm{Cl}^{-}$, TSH, ACTH, PRL and $\mathrm{GH}$ ) were compared between the groups with the difference value (D-value) of the blood index prior to and following the operation.

Detection of residual ${ }^{32} P$ deposition. Venous blood $(2 \mathrm{ml})$ and urine $(2 \mathrm{ml})$ were obtained from all patients at 1, 3 and 7 days post-surgery. Radioactive counts per minute (CPM) within each sample were quantitatively measured using the CAPRAC well-type $\mathrm{NaI} \gamma$ counter to evaluate ${ }^{32} \mathrm{P}$ deposition.

Statistical analysis. SPSS software (version 17; SPSS, Inc., Chicago, IL, USA) was used for data analysis. Results are presented as mean \pm standard deviation. Data were analyzed for normality, and an analysis of variance for randomized block design was performed. The Student-Newman-Keuls test was then utilized for group comparisons and Dunnett's test was used to analyze blood and urine CPM values between each study group and the negative control group (normal blood and urine specimens). An analysis of variance and Bonferroni's correction were used to analyze leakage rate. $\mathrm{P}<0.05$ was considered to indicate a statistically significant difference.

\section{Results}

Comparison of general data between study groups. No significant differences were identified between patients $(n=40)$ 
with regard to the general condition (age, weight and surgical duration; P>0.05; Table I).

Medical history. In total, 24 patients had a history of previous CP resection, 29 underwent internal radiotherapy with ${ }^{32} \mathrm{P}$ colloid for $\mathrm{CP}$ following stereotactic aspirations of cystic fluid, 11 underwent stereotactic aspiration alone, 7 accepted $\gamma$-knife treatment, 4 were treated with a ventriculoperitoneal shunt and 2 were implanted with Ommaya reservoirs.

Preoperative signs, symptoms and laboratory results. The primary clinical symptoms regarding intracranial hypertension included headache, nausea and vomiting in 18 patients, and polydipsia and polyuria in 12 patients. Vision loss was identified in 34 patients (4 with monocular blindness), with varying degrees of visual field defects. Developmental disorders, which were short and without the development of secondary sexual characteristics, were observed in 4 patients. Abnormal pre-surgery levels were observed for the following hormones: TSH ( $n=10$; the normal range values, 0.49-4.91 mIU/l), ACTH $(\mathrm{n}=22$; the normal range values, 7.2-63.3 pg/ml), LH $[\mathrm{n}=1$; the normal range values, period of follicular (2.22-10.9 IU/1), period of ovulation (19.1-103 IU/1), period of luteal (1.2-12.9 IU/1), period of the menopause (10.9-58.6 IU/1)], PRL $(n=5$; the normal range values, $70.8-566.5 \mathrm{mIU} / \mathrm{l})$ and $\mathrm{GH}(\mathrm{n}=4$; the normal range values, $0-5 \mathrm{ng} / \mathrm{ml})$.

Clinical efficacy. Surgery was successful for all patients; the volume of the capsule was reduced, the injection was successful, and the postoperative symptoms were relieved without complications. Overall, 1 patient formed a small epidural hematoma, which was treated using the conservative regimen (hemostasis, dehydrated drugs and symptomatic treatment); 1 patient suffered from a small amount of bleeding from the frontal lobe puncture path, which was improved following the conservative regimen; 19 patients exhibited small amounts of intracranial gas accumulation, which were self-absorbed; the cystic volume was significantly decreased in 37 patients post-surgery; hormone levels returned to the normal reference range post-surgery in 5 patients ( $\mathrm{TSH}, \mathrm{n}=2$; PRL, $\mathrm{n}=2$ and $\mathrm{GH}$, $\mathrm{n}=1$ ), whereas 3 patients (TSH, $\mathrm{n}=2$ and $\mathrm{ACTH}, \mathrm{n}=1$ ) exhibited abnormal hormone levels, and no other marked alterations were observed. Improvement in vision was observed in 21 patients following surgery and no other marked changes were observed during ophthalmic examination. No wound or intracranial infection was observed following surgery. Results demonstrated significant differences between the predicted cystic cavity volume prior to surgery and the cystic fluid volume aspirated during surgery among different dose groups $(\mathrm{P}<0.05$; Table II).

Drug distribution and leakage. Head CT results obtained from all patients $2 \mathrm{~h}$ after surgery demonstrated that heterogeneous drug and contrast agent distributions were present in 6 patients within the $2.0 \mathrm{mCi}$ group, 2 patients within the $1.5 \mathrm{mCi}$ group and 2 patients within the $1.0 \mathrm{mCi}$ group. This was due to the fact that the ventricles were inevitably involved in the puncture routes, resulting in shrinkage of the cavity volume following the intracavitary CSF leakage (Fig. 1A-C). However, the remaining patients demonstrated that the drug and the contrast agent were heterogeneously distributed and
Table I. Comparison between groups with regard to the general condition of each patient (mean \pm standard deviation).

\begin{tabular}{lcccc}
\hline Group & $\mathrm{n}$ & Age, years & Weight, kg & $\begin{array}{c}\text { Surgical } \\
\text { duration, } h\end{array}$ \\
\hline $0.5 \mathrm{mCi}$ & 10 & $31.10 \pm 18.41$ & $60.50 \pm 16.43$ & $1.59 \pm 0.50$ \\
$1.0 \mathrm{mCi}$ & 10 & $30.10 \pm 18.07$ & $58.80 \pm 17.50$ & $1.64 \pm 0.37$ \\
$1.5 \mathrm{mCi}$ & 10 & $32.20 \pm 20.23$ & $61.50 \pm 19.86$ & $1.62 \pm 0.40$ \\
$2.0 \mathrm{mCi}$ & 10 & $29.60 \pm 19.25$ & $62.90 \pm 17.04$ & $1.57 \pm 0.45$ \\
\hline
\end{tabular}

No significant differences were identified between groups regarding general conditions $(\mathrm{P}>0.05)$.

Table II. Comparison of each patient's cystic cavity volume, extraction fluid volume and leakage rate between groups prior to surgery (mean \pm standard deviation).

\begin{tabular}{lcccc}
\hline Group & $\mathrm{n}$ & $\begin{array}{c}\text { Leakage } \\
\text { rate, } \%\end{array}$ & $\begin{array}{c}\text { Predicted cystic } \\
\text { cavity } \\
\text { volume, ml }\end{array}$ & $\begin{array}{c}\text { Extracted } \\
\text { cystic fluid } \\
\text { volume, ml }\end{array}$ \\
\hline $0.5 \mathrm{mCi}$ & 10 & 0 & $3.54 \pm 0.66$ & $3.46 \pm 0.64$ \\
$1.0 \mathrm{mCi}$ & 10 & 10 & $7.44 \pm 1.08^{\mathrm{a}}$ & $7.12 \pm 1.35^{\mathrm{a}}$ \\
$1.5 \mathrm{mCi}$ & 10 & 20 & $12.29 \pm 1.84^{\mathrm{a}, \mathrm{b}}$ & $12.79 \pm 1.47^{\mathrm{a}, \mathrm{b}}$ \\
$2.0 \mathrm{mCi}$ & 10 & $60^{\mathrm{a}, \mathrm{b}}$ & $16.84 \pm 1.01^{\mathrm{a}, \mathrm{b}, \mathrm{c}}$ & $16.95 \pm 1.51^{\mathrm{a}, \mathrm{b}, \mathrm{c}}$ \\
\hline
\end{tabular}

${ }^{\mathrm{a}} \mathrm{P}<0.05$ compared with the $0.5 \mathrm{mCi}$ group; ${ }^{\mathrm{b}} \mathrm{P}<0.05$ compared with the $1.0 \mathrm{mCi}$ group; and ${ }^{\mathrm{c}} \mathrm{P}<0.05$ compared with the $1.5 \mathrm{mCi}$ group.

no leakage was identified. Therefore, cavity volumes were significantly decreased (Fig. 1D-F) and the leakage rate was significantly increased between the $2.0 \mathrm{mCi}$ group and the 1.0 and $0.5 \mathrm{mCi}$ groups (both $\mathrm{P}<0.05$; Table II).

\section{Drug safety}

Comparison of hematological parameters prior to and following surgery among different groups. No significant differences were identified in group comparisons between blood routine results, blood biological parameters and pituitary hormone levels among 24 patients pre-surgery and at 7 days post-surgery ( $\mathrm{P}>0.05$; Table III). Prior to surgery, blood routine results and blood biological parameters from all the patients were within the normal reference ranges. However, within the $2.0 \mathrm{mCi}$ group, two patients presented with increased ALT (the normal range values, 5-35 U/l) levels, one exhibited decreased levels of $\mathrm{K}^{+}$(the normal range values, 3.5-5.3 mmol/l), $\mathrm{Na}^{+}$(the normal range values, $136-145 \mathrm{mmol} / \mathrm{l}$ ), and $\mathrm{Cl}^{-}$(the normal range values, 96-108 $\mathrm{mmol} / \mathrm{l}$ ) and one demonstrated a mildly prolonged coagulation time following surgery, while in the $1.0 \mathrm{mCi}$ group, one patient demonstrated decreased levels of $\mathrm{K}^{+}, \mathrm{Na}^{+}$and $\mathrm{Cl}^{-}$following surgery. Abnormal pituitary hormone levels were identified in patients in different groups prior to surgery: 3 patients demonstrated decreased TSH levels, 2 demonstrated abnormally decreased ACTH levels, 1 demonstrated slightly increased PRL levels 
Table III. D-value results of main blood test indices for differences prior to and following surgery for each group (mean \pm standard deviation).

\begin{tabular}{lcccccccccc}
\hline Group & $\mathrm{n}$ & ALT, U/l & AST, U/l & $\begin{array}{c}\mathrm{K}^{+}, \\
\mathrm{mmol} / 1\end{array}$ & $\begin{array}{c}\mathrm{Na}^{+}, \\
\mathrm{mmol} / 1\end{array}$ & $\begin{array}{c}\mathrm{Cl}^{-}, \\
\mathrm{mmol} / 1\end{array}$ & $\begin{array}{c}\mathrm{TSH}, \\
\mathrm{mIU} / \mathrm{l}\end{array}$ & $\begin{array}{c}\mathrm{ACTH}, \\
\mathrm{pg} / \mathrm{ml}\end{array}$ & $\begin{array}{c}\mathrm{PRL}, \\
\mathrm{IU} / \mathrm{l}\end{array}$ & $\begin{array}{c}\mathrm{GH}, \\
\mathrm{ng} / \mathrm{ml}\end{array}$ \\
\hline $0.5 \mathrm{mCi}$ & 8 & $-0.74 \pm 1.77$ & $-0.78 \pm 1.65$ & $0.61 \pm 0.32$ & $6.13 \pm 4.04$ & $5.22 \pm 3.82$ & $0.08 \pm 0.05$ & $2.35 \pm 0.99$ & $2.79 \pm 1.64$ & $0.01 \pm 0.01$ \\
$1.0 \mathrm{mCi}$ & 8 & $-0.96 \pm 1.78$ & $-0.97 \pm 1.67$ & $0.69 \pm 0.47$ & $5.79 \pm 4.90$ & $5.37 \pm 3.71$ & $0.09 \pm 0.05$ & $2.12 \pm 1.30$ & $2.69 \pm 2.04$ & $0.01 \pm 0.01$ \\
$2.0 \mathrm{mCi}$ & 8 & $0.08 \pm 4.68$ & $0.11 \pm 4.44$ & $0.69 \pm 0.80$ & $6.90 \pm 4.83$ & $5.41 \pm 3.94$ & $0.09 \pm 0.07$ & $2.32 \pm 1.81$ & $2.32 \pm 2.96$ & $0.01 \pm 0.01$ \\
\hline
\end{tabular}

Main blood indices prior to and following surgery demonstrated no significant differences between groups ( $\mathrm{P}>0.05)$. ALT, aspartate aminotransferase; AST, alkaline phosphatase; $\mathrm{K}^{+}$, potassium; $\mathrm{Na}^{+}$, sodium; $\mathrm{Cl}^{-}$, chloride; TSH, thyroid-stimulating hormone; ACTH, adrenocorticotropic hormone; PRL, prolactin; GH, growth hormone.

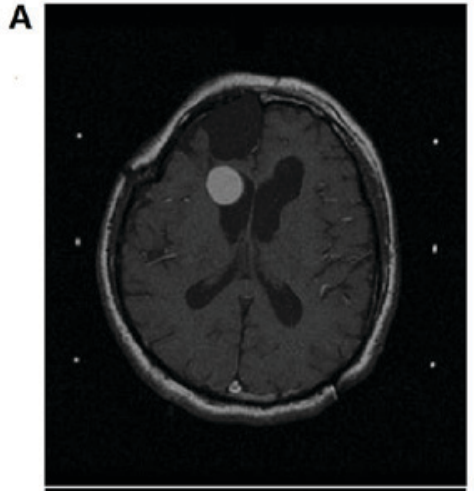

D

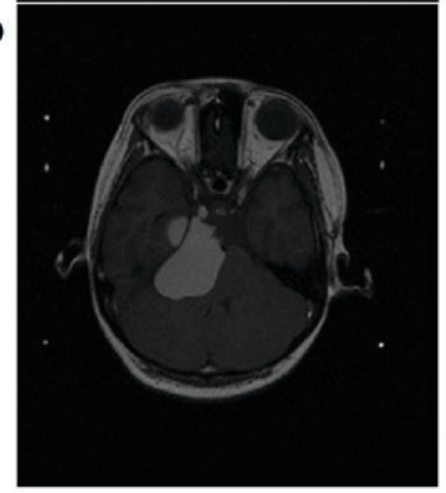

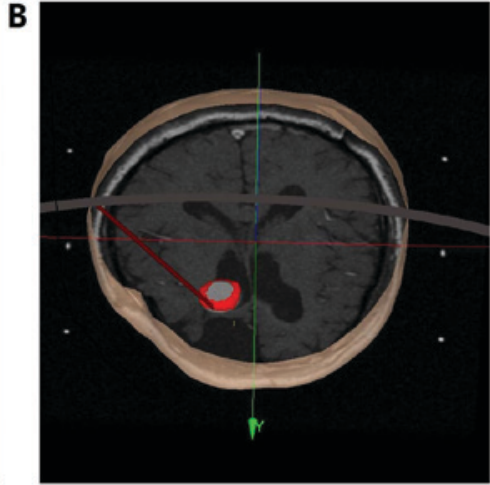

E

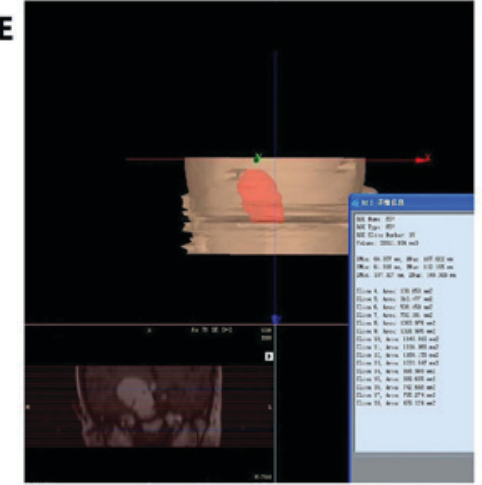

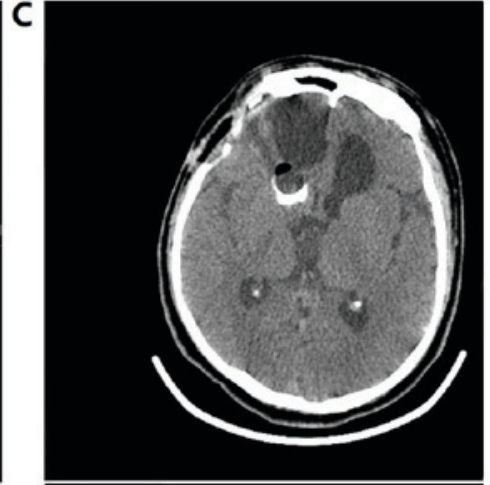

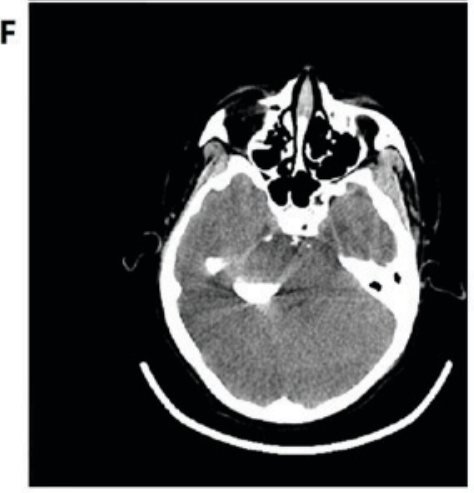

Figure 1. (A-C) Imaging results obtained pre- and post-surgery from a patient with CSF leakage whose puncture route failed to avoid the ventricles. (A) Positioning head MRI scan (prior to surgery), indicating a close association between the CP cyst and the ventricle. (B) Puncture route planned by the stereotactic surgery planning system. (C) Image obtained from the head CT scan performed $1 \mathrm{~h}$ after surgery in order to evaluate the contrast agent distribution and leakage. Image demonstrates an intracavitary heterogeneous enhancement and unchanged cavity size, with a small amount of gas accumulation. (D-F) Imaging results obtained pre- and post-surgery from a patient without CSF leakage. (D) Positioning head MRI scan (prior to surgery), indicating a close association between the CP cyst and the pons. (E) Estimated cystic fluid volume of $\sim 33 \mathrm{ml}$ and the puncture route planned by the stereotactic surgery planning system, which successfully avoided the ventricle. (F) Image generated from the head CT scan performed $1 \mathrm{~h}$ after surgery demonstrating intracavitary homogeneous enhancement and decreased cavity size without gas accumulation. CSF, cerebrospinal fluid; MRI, magnetic resonance imaging; CP, craniopharyngioma; CT, computed tomography.

and 1 demonstrated decreased $\mathrm{GH}$ levels in the $2.0 \mathrm{mCi}$ group, whereas 1 patient demonstrated decreased TSH levels, 1 demonstrated decreased ACTH levels and 1 demonstrated decreased $\mathrm{GH}$ levels in the $1.0 \mathrm{mCi}$ group. Abnormal postoperative pituitary hormone levels were also identified in patients in different groups: In the $2.0 \mathrm{mCi}$ group, 2 patients exhibited decreased TSH levels, 2 exhibited decreased ACTH levels and 1 exhibited decreased $\mathrm{GH}$ levels, whereas in the $1.0 \mathrm{mCi}$ group, 1 patient exhibited decreased TSH levels and 1 patient exhibited decreased ACTH levels. In the $0.5 \mathrm{mCi}$ group, 1 patient demonstrated decreased ACTH levels following surgery. Although abnormal variations were observed prior to and following surgery in certain individual surgical indices, no significant differences were identified when compared with major hematological parameters (ALT, AST, $\mathrm{K}^{+}, \mathrm{Na}^{+}, \mathrm{Cl}^{-}, \mathrm{TSH}$, $\mathrm{ACTH}, \mathrm{PRL}$ and $\mathrm{GH}$ ) from each group prior to and 7 days after surgery $(\mathrm{P}>0.05)$.

Residue ${ }^{32} \mathrm{P}$ depositions among different groups. Venous blood $(2 \mathrm{ml})$ and urine $(2 \mathrm{ml})$ were obtained from 24 patients at 1, 3 and 7 days post-surgery. The CAPRAC well-type $\mathrm{NaI} \gamma$ counter was used to measure the CPM and blank controls were set accordingly. Results indicated a significant difference in blood CPM values 1 day after surgery and in urine 

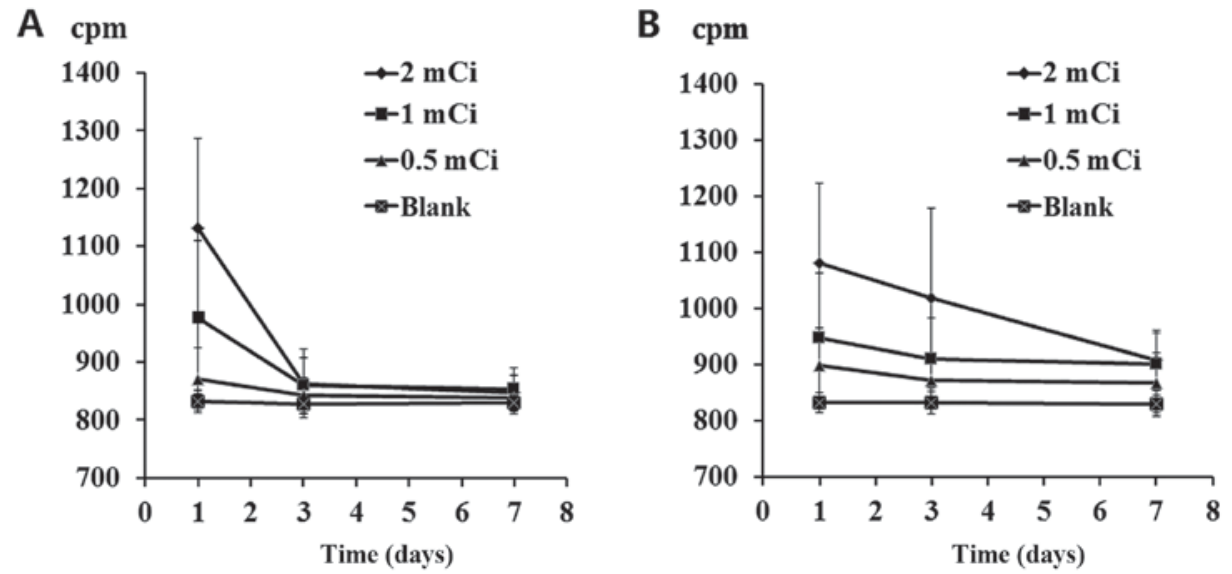

Figure 2. Alterations in radioactive phosphorus-32 colloid CPM identified in (A) blood and (B) urine samples from each group within 7 days after surgery. CPM, counts per minute.

CPM values 1,3 and 7 days after surgery between the 2.0 and $1.0 \mathrm{mCi}$ groups and the control group $(\mathrm{P}<0.05)$. Blood deposition returned to normal within 3 days, whereas urine deposition returned to normal in $\sim 7$ days (Fig. 2).

\section{Discussion}

The present study successfully investigated the distribution and leakage of ${ }^{32} \mathrm{P}$ injected into the $\mathrm{CP}$ cystic cavity using the contrast agent developing technology (a head CT scan to check the iopamidol-300 has been injected into the CP cystic cavity). This method, compared with the single-photon emission computed tomography (CT) imaging (8), is advantageous with regard to convenience, immediacy and low cost. Head CT scans, which were performed following surgery, indicated the accumulation of a small amount of intracavitary gas in the majority of patients. Negative pressure caused by cyst fluid aspiration may have contributed to the accumulation and these small amounts of gas may have entered the cyst at the time of syringe replacement and/or drug injection. Despite gas being self-absorbed in the long term, the intracranial gas accumulation can still be avoided using a T-tube to improve the injection process and withdrawing the puncture needle directly following the completion of the injection. The primary reason for the heterogeneous $\mathrm{CT}$ images was due to negative pressure being created by the connection between the puncture route and the ventricle, this caused the CSF to pass through the puncture hole in the $\mathrm{CP}$ wall and enter into the cystic cavity, which diluted the contrast agent and the ${ }^{32} \mathrm{P}$ colloid. As the target is typically set at the center of the cystic cavity, imaging results demonstrated a CSF flushing dilution zone within the cavity center. In such cases, when the cystic fluid is aspirated during the surgery, the fluid color becomes gradually clear and the negative pressure is removed. When the aspirated fluid volume is significantly increased compared with the pre-surgical estimate, CSF leakage occurs and the aspiration is terminated immediately. Therefore, with the premise of ensuring a safe intracranial puncture route, the ventricle system should be maximally avoided during the pre-surgical route planning. Usually, when the route is not connected to the ventricular system, negative pressure increases as the aspiration continues. Negative pressure appears when the fluid is completely aspirated, and therefore, further aspiration should be avoided. In these cases, a significantly decreased cavity size with homogeneous enhancement of contrast agent is observed in the head CT scans following surgery. A difference between the preoperative estimated cystic fluid volume and the actual aspirated cystic fluid volume was investigated in the present study. The differences observed may be associated with the tracing accuracy of the surgery planning system for each cyst cross-section presented on the MRI prior to surgery, the degree of aspiration, the amount of CSF leakage and the presence of multiple CP cysts, as well as whether they were interconnected. Furthermore, the degree of cyst wall calcification also affects the planning for the puncture route, whereas the elastic retraction degree of the cyst wall also determines the amount of fluid aspiration and the degree of cystic cavity shrinkage. Overall, as patient $\mathrm{CP}$ cystic cavity volume increased, the volume of required ${ }^{32} \mathrm{P}$ colloid also increased [calculated based on the equation (2)]. Patients who required an increased volume of ${ }^{32} \mathrm{P}$ colloid also required a more complex intracranial puncture route in order to avoid the ventricle containing part of the protruding cyst wall(s), therefore, this increased the risk of heterogeneous drug and contrast agent distribution due to CSF leakage. However, it is important to note that even if the imaging route plan avoids the ventricle system, CSF leakage may still occur during surgery.

The present study also investigated the deposition, excretion, toxicity and side effects of the ${ }^{32} \mathrm{P}$ colloid following surgery. Surgery was successful in all patients, and according to the comparison of clinical and imaging results, the effectiveness (classified as the volume of the capsule being reduced, the injection was successful, and the postoperative symptoms were relieved without complications) was $100 \%$; however, certain patients still suffered from poorly improved vision, visual field and hormone levels, whereas other individuals exhibited mildly decreased hormone levels. Patients who demonstrated a decrease in hormone levels were administered hormone supplements (including thyroxine tablets, dexamethasone tablets and ambroxol tablets) and regular follow-ups were performed. All patient hormone levels returned to their respective normal reference range and the side effects caused by hormone supplements were effectively managed. Results obtained from imaging analysis demonstrated a 
slight decrease in $\mathrm{CP}$ cystic volume in certain individuals, indicating the presence of CSF leakage from the intracranial puncture route. Therefore, disease observation, blood tests and imaging examination during the follow-up should be provided to these patients. Intraventricular leakage of ${ }^{32} \mathrm{P}$ colloid is occasionally inevitable and the drug spreads along the CSF circulation pathway following entrance into the ventricular system, causing further radioactive damage to several types of neurological system, including the hypothalamus. Despite comparison of hematological parameters prior to and following surgery, results demonstrated no significant different in any group; however slightly elevated transaminase levels, mild coagulation abnormalities and electrolyte imbalance occurred in certain patients. A previous study (9) demonstrated that ${ }^{32} \mathrm{P}$ colloid caused coagulation and liver dysfunction due to a decrease in antiplatelets; however, these dysfunctions were completely restored on their own accord following 8 weeks. Therefore, monitoring hematological parameters within 1 month after surgery is of high importance. As the duration of hospitalization is usually $<7$ days, it is essential to increase the monitoring of each hematological parameter and symptom following patient discharge. In the present study, time points for measuring CPM following surgery in blood and urine samples were rather limited and also all within 1 week, whereas the half-life of ${ }^{32} \mathrm{P}$ is $\sim 2$ weeks and the excretion cycle is $>2$ weeks. Therefore, the follow-up duration following discharge should be prolonged, or preferably be replaced with continuous monitoring until the CPM value has returned to normal. Due to the limited research in this area, the complete metabolic pathway of ${ }^{32} \mathrm{P}$ colloid remains unclear. The absorbance of ${ }^{32} \mathrm{P}$ colloid into the blood through the cyst wall prior to being decomposed by the liver requires investigation. However, it is important to note that discharged ${ }^{32} \mathrm{P}$ colloid only accounts for a small proportion of the total injected dose and that the majority of the drug is still distributed and deposited into the $\mathrm{CP}$ cystic cavity until it loses its radioactivity; however, further investigation into the presence of residue in feces and sweat excretion are required.

It important to recognize the limitations of the present study. The long-term distribution and leakage of ${ }^{32} \mathrm{P}$ colloid could not be monitored continuously and further measurements of ${ }^{32} \mathrm{P}$ colloid radioactivity inside the cyst and/or CSF fluid were not provided. Additionally, the enrolled patients should have been followed up in order to determine long-term efficacy. Furthermore, surgical manipulations and route planning affected the present results and surgical efficacy to varying degrees. Associated factors which influenced results included the surgeon's surgical habits (artificial differences in surgical software planning, artificial control differences in cystic fluid aspiration level, artificial selection of puncture needle, surgical technique difference, etc.), estimations of the cystic volume and drug doses, accuracy of the evaluation for visual recovery and symptom improvement, intraoperative judgment for the amount and degree of cyst fluid aspiration, and the patient's tolerance to the local anesthesia and surgery. The CAPRAC well-type NaI $\gamma$ counter was used to quantitatively measure the ${ }^{32} \mathrm{P}$ colloid radioactive CPM in the patients' blood and urine, and to evaluate the presence of residual ${ }^{32} \mathrm{P}$ colloid deposition. This method, compared with the previous liquid scintillation counter (10), is advantageous with regard to convenience, immediacy and low cost during radioactive counting, therefore, it is suitable for the continuous monitoring of a patient's residual ${ }^{32} \mathrm{P}$ colloid deposition. The present study provides a method for evaluating the distribution of ${ }^{32} \mathrm{P}$ colloid radiotherapy for craniopharyngioma and proves its safety and efficacy.

\section{Acknowledgements}

Not applicable.

\section{Funding}

The present study was supported by the Innovation and Development Foundation of the Navy General Hospital.

\section{Availability of data and materials}

The datasets used and/or analyzed during the current study are available from the corresponding author on reasonable request.

\section{Authors' contributions}

$\mathrm{HC}$ and $\mathrm{JZ}$ designed the experiment, $\mathrm{HC}, \mathrm{WC}, \mathrm{YW}, \mathrm{HZ}$ and RL performed the experiment, $\mathrm{HC}$ and SG processed the data, $\mathrm{HC}$ wrote and edited the paper.

\section{Ethics approval and consent to participate}

Written informed consent was obtained from either the patient or a relative, and the present study was approved by the Ethics Committee of the Navy General Hospital.

\section{Consent for publication}

Written informed consent was obtained from either the patient or a relative.

\section{Competing interests}

The authors declare that they have no competing interests.

\section{References}

1. Liubinas SV, Munshey AS and Kaye AH: Management of recurrent craniopharyngioma. J Clin Neurosci 18: 451-457, 2011.

2. Taasan V, Shapiro B, Taren JA, Beierwaltes WH, McKeever P, Wahl RL, Carey JE, Petry N and Mallette S: Phosphorus-32 therapy of cystic Grade IV astrocytomas: Technique and preliminary application. J Nucl Med 26: 1335-1338, 1985.

3. Hasegawa T, Kondziolka D, Hadjipanayis CG and Lunsford LD: Management of cystic craniopharyngiomas with phosphorus-32 intracavitary irradiation. Neurosurgery 54: 813-820, 2004.

4. Kickingereder P, Maarouf M, El Majdoub F, Fuetsch M, Lehrke R, Wirths J, Luyken K, Schomaecker K, Treuer H, Voges J and Sturm V: Intracavitary brachytherapy using stereotactically applied phosphorus-32 colloid for treatment of cystic craniopharyngiomas in 53 patients. J Neurooncol 109: 365-374, 2012.

5. Tian Z, Liu Z and Wang Y: Stereotactic intratumoral irradiation of huge craniopharyngioma. Zhonghua Zhong Liu Za Zhi 18: 234-236, 1996 (In Chinese).

6. Yu X, Zhang JN, Liu R, Wang YM, Sun JZ, Qi SB, DU YN Wang HW, Zhao HL and Liu ZH: Mixed craniopharyngioma: Long-term results after gamma knife combined with stereotactic brachytherapy. Zhonghua Wai Ke Za Zhi 51: 631-635, 2013 (In Chinese). 
7. Trippel M and Nikkhah G: Stereotactic neurosurgical treatment options for craniopharyngioma. Front Endocrinol (Lausanne) 3: 63, 2012.

8. Denis-Bacelar AM, Romanchikova M, Chittenden S, Saran FH, Mandeville H, Du Y and Flux GD: Patient-specific dosimetry for intracavitary ${ }^{32} \mathrm{P}$-chromic phosphate colloid therapy of cystic brain tumours. Eur J Nucl Med Mol Imaging 40: 1532-1541, 2013

9. Liu L, Teng G, Zhang D, Song J, He S, Guo J and Fang W: Toxicology of intrahepatic arterial administration of interventional phosphorus-32 glass microspheres to domestic pigs. Chin Med J (Engl) 112: 632-636, 1999.
10. Gaca P, Warwick PE and Croudace IW: Liquid scintillation counters calibration stability over long timescales. J Radioanal Nucl Chem 314: 753-760, 2017.

(i) (5) This work is licensed under a Creative Commons Attribution-NonCommercial-NoDerivatives 4.0 International (CC BY-NC-ND 4.0) License. 